\title{
Application of Hypergeometric Series in the Inverse Moments of Special Discrete Distribution*
}

\author{
Hongyu Bao, Wuyungaowa \\ Department of Mathematics, College of Sciences and Technology, Inner Mongolia University, Huhhot, China \\ Email: 1486373541@qq.com
}

How to cite this paper: Bao, H.Y. and Wuyungaowa (2017) Application of Hypergeometric Series in the Inverse Moments of Special Discrete Distribution. Journal of Applied Mathematics and Physics, 5, 267275.

https://doi.org/10.4236/jamp.2017.52024

Received: December 29, 2016

Accepted: February 12, 2017

Published: February 15, 2017

\begin{abstract}
In this paper, we use the generalized hypergeometric series method the highorder inverse moments and high-order inverse factorial moments of the generalized geometric distribution, the Katz distribution, the Lagrangian Katz distribution, generalized Polya-Eggenberger distribution of the first kind and so on.
\end{abstract}

\section{Keywords}

Hypergeometric Series, Inverse Moments, Factorial Inverse Moments

\section{Introduction}

The moment is one of the most widely used features of probability of random variables. The moments of random variables have been widely used in many important fields such as finance, probability theory, statistics and so on. So the calculation of the moment is very important. The inverse moment is a hot research direction in recent years. Inverse moment plays an important role in risk assessment, insurance and finance, and it is an important concept in probability. The study of the inverse moments originates from random sampling, $x$ is the number of observations $z_{1}, z_{2}, \cdots z_{x}$ with mean $Z=\frac{z_{1}, z_{2}, \cdots z_{x}}{x}$ if $z_{i}(i=1,2 \cdots x)$ is independent and identically distributed random variable, the variance is $\sigma^{2}$,when $x$ is a constant, the variance of $z$ is $\frac{\sigma^{2}}{x}$, but when the $x$ is a random variable, the variance of $z$ was $\sigma^{2} E\left(\frac{1}{x}\right)$, at this point in the

${ }^{*}$ Funded projects: The research is supported by the National Natural Science Foundation of China under Grant. 
sampling problem of inverse moment are introduced. Generally, the distribution of $x$ is mainly the Poisson distribution, binomial distribution and so on.

The research on inverse moments of the binomial distribution and the Poisson distribution has been a long history. In 1945, Frederick F. Stephan studied the inverse moments of first and second order of the binomial distribution (see [1]). Grab and Stephan calculated tables of reciprocals for binomial and Poisson distribution as well as derive a recurrence relation. They also derived an exact expression for the first inverse moment (see [2]). Govindarajulu in 1963 a recursive formula moments of binomial distribution has been obtained (see [3]). In 1972, Chao and Strawderman (see [4]) considered slightly different inverse moments defined as $E\left(\frac{1}{(x+a)^{r}}\right)$ which are frequently easier to calculate.

At present, more and more scholars are interested in the study of inverse moment, and have a wealth of research results mainly binomial distribution, Poisson distribution, negative binomial distribution, logarithmic distribution (see [5]). In this paper describes the use of generalized hypergeometric series inverse moments and factorial inverse moment distribution of some. It mainly includes Janardan discussed the distribution of the generalized Polya-Eggenberger distribution of the first kind, and the special value of the parameters (see [6]).

In the next, we will give some definitions necessarily.

Definition 1: Suppose $X$ is a generalized geometric random variable with parameters $\lambda$, having probability mass function

$$
P_{x}(\lambda)=x \lambda^{x-1}(1-\lambda)^{2}, x=1,2, \cdots,
$$

where $0<\lambda<1$

Definition 2: Suppose $X$ is a generalized Polya-Eggenberger of the first kind random variable with parameters $a, b, c, \beta$ having probability mass function

$$
P_{x}(a, \beta)=\frac{\frac{a}{c}}{\frac{a+x b}{c}+x}\left(\begin{array}{c}
\frac{a+x b}{c}+x \\
x
\end{array}\right) \beta^{x}(1-\beta)^{\frac{a+x b}{c}}, x=0,1,2, \cdots,
$$

where $a>0, c>0, b+c \geq 0,0<\beta<1$.

Definition 3: Suppose $\mathrm{X}$ is a Katz random variable with parameters $a, \beta$, having probability mass function

$$
P_{x}(a, \beta)=\left(\begin{array}{c}
\frac{a}{\beta}+x-1 \\
x
\end{array}\right) \beta^{x}(1-\beta)^{\frac{a}{\beta}}, x=0,1,2, \cdots,
$$

where $a>0,0<\beta<1$.

Definition 4: Suppose $X$ is a Lagrangian Katz random variable with parameters $a, b, \beta$, having probability mass function

$$
P_{x}(a, \beta)=\frac{\frac{a}{\beta}}{\frac{a}{\beta}+\frac{x b}{\beta}+x}\left(\begin{array}{c}
\frac{a}{\beta}+\frac{x b}{\beta}+x \\
x
\end{array}\right) \beta^{x}(1-\beta)^{\frac{a}{\beta}+\frac{x b}{\beta}}, x=0,1,2, \cdots,
$$


where $a>0, b+\beta \geq 0,0<\beta<1$.

The definition of generalized hypergeometric series:

$$
\begin{gathered}
{ }_{p} F_{q}\left[\left(a_{1}, k\right),\left(a_{2}, k\right), \cdots,\left(a_{p}, k\right) ;\left(b_{1}, k\right)\left(b_{2}, k\right), \cdots,\left(b_{q}, k\right) z\right] \\
=\sum_{n=0}^{\infty} \frac{\left(a_{1}\right)_{n}^{k}\left(a_{2}\right)_{n}^{k} \cdots\left(a_{p}\right)_{n}^{k} z^{n}}{\left(b_{1}\right)_{n}^{k}\left(b_{2}\right)_{n}^{k} \cdots\left(b_{q}\right)_{n}^{k} n !}=1+\frac{a_{1}^{k} \cdot a_{2}^{k} \cdots a_{p}^{k}}{b_{1}^{k} \cdot b_{2}^{k} \cdots b_{q}^{k}} z \\
+\frac{\left[a_{1}\left(a_{1}+1\right)\right]^{k}\left[a_{2}\left(a_{2}+1\right)\right]^{k} \cdots\left[a_{p}\left(a_{p}+1\right)\right]^{k} z^{2}}{\left[b_{1}\left(b_{1}+1\right)\right]^{k}\left[b_{2}\left(b_{2}+1\right)\right]^{k} \cdots\left[b_{q}\left(b_{q}+1\right)\right]^{k} 2 !}+\cdots
\end{gathered}
$$

where $\left(\lambda_{i}\right)_{n}^{k}=\left(\lambda_{i}\right)^{k}\left(\lambda_{i}+1\right)^{k}\left(\lambda_{i}+2\right)^{k} \cdots\left(\lambda_{i}+n-1\right)^{k},\left(\lambda_{i}\right)_{0}^{k}=1, i=1,2, \cdots, p$.

$$
\text { If } k=1 \text { then }{ }_{p} F_{q}=\sum_{n=0}^{\infty} \frac{\left(a_{1}\right)_{n}\left(a_{2}\right)_{n} \cdots\left(a_{p}\right)_{n}}{\left(b_{1}\right)_{n}\left(b_{2}\right)_{n} \cdots\left(b_{q}\right)_{n}} \frac{z^{n}}{n !} \text {. is hypergeometric series. }
$$

\section{The Inverse Moments of Some Discrete Distributions}

In this section, we use a generalized hypergeometric series to obtain the inverse moments of some discrete distributions.

Theorem 2.1: Suppose $x$ is a generalized geometric random variable with parameters $\lambda$, for $0<\lambda<1$, then the inverse moment of $k^{\text {th }}$ order is given by

$$
E(X+A)^{-k}=\frac{(1-\lambda)^{2}}{(A+1)^{k}}{ }_{2} F_{1}[(A+1, k), 2 ;(A+2, k) ; \lambda]
$$

where $A \geq 0$.

Proof. By definition 1, then

$$
\begin{aligned}
E(X+A)^{-k} & =\sum_{x=1}^{\infty} x \lambda^{x-1}(1-\lambda)^{2} \frac{1}{(x+A)^{k}} \\
& =\frac{(1-\lambda)^{2}}{(A+1)^{k}}+\frac{2 \lambda(1-\lambda)^{2}}{(A+2)^{k}}+\frac{3 \lambda^{2}(1-\lambda)^{2}}{(A+3)^{k}}+\cdots \\
& =\frac{(1-\lambda)^{2}}{(A+1)^{k}}\left\{1+\frac{2(A+1)^{k}}{(A+2)^{k}} \frac{\lambda}{1 !}+\frac{2 \cdot 3(A+1)^{k}(A+2)^{k}}{(A+2)^{k}(A+3)^{k}} \frac{\lambda^{2}}{2 !}+\cdots\right\} \\
& =\frac{(1-\lambda)^{2}}{(A+1)^{k}} \sum_{n=0}^{\infty} \frac{(A+1)_{n}^{k}(2)_{n}}{(A+2)_{n}^{k}} \cdot \frac{\lambda^{n}}{n !} \\
& =\frac{(1-\lambda)^{2}}{(A+1)^{k}}{ }_{2} F_{1}[(A+1, k), 2 ;(A+2, k) ; \lambda] .
\end{aligned}
$$

Note: when $k=1$, the inverse moment of first order is given by

$$
E(X+A)^{-1}=\frac{(1-\lambda)^{2}}{A+1}{ }_{2} F_{1}[A+1,2 ; A+2 ; \lambda]
$$

Theorem 2.2: Suppose $x$ is a generalized Polya-Eggenberger of the first kind random variable with parameters $a, b, c, \beta$, for $a>0, c>0, b+c \geq 0,0<\beta<1$. then we have the inverse moment of $k^{\text {th }}$ order is given by 


$$
\begin{aligned}
& E(X+A)^{-1} \\
& =\frac{\frac{a}{c} \beta(1-\beta)^{\frac{a+b}{c}}}{(A+1)^{k}}{ }_{3} F_{21}\left[(A+1),\left(\frac{a+(n+1) b+c}{c}\right), 1 ;(A+2, k), 2 ; \beta(1-\beta)^{\frac{b}{c}}\right]
\end{aligned}
$$

where $A \geq 0, n=0,1,2 \cdots$

Proof. By definition 2, then

$$
\begin{aligned}
& E(X+A)^{-k}=\sum_{x=1}^{\infty} \frac{a}{c} \frac{1}{\left(\frac{a+x b}{c}+x\right)}\left(\frac{a+x b}{c}+x\right) \beta^{x}(1-\beta)^{\frac{a+x b}{c}} \frac{1}{(x+A)^{k}} \\
= & \sum_{x=1}^{\infty} \frac{a}{c} \frac{\left(\frac{a+x b}{c}+x-1\right) !}{x !\left(\frac{a+x b}{c}\right) !} \beta^{x}(1-\beta)^{\frac{a+x b}{c}} \frac{1}{(x+A)^{k}} \\
= & \frac{a}{c} \beta(1-\beta)^{\frac{a+b}{c}} \frac{1}{(A+1)^{k}}\left\{1+\frac{(A+1)^{k}\left(\frac{a+2 b}{c}+1\right)}{(A+2)^{k} \cdot 2 !} \beta(1-\beta)^{\frac{b}{c}}\right. \\
& \left.+\frac{(A+1)^{k}\left(\frac{a+3 b}{c}+1\right)\left(\frac{a+3 b}{c}+2\right)}{(A+3)^{k} \cdot 3 !} \beta^{2}(1-\beta)^{\frac{2 b}{c}}+\cdots\right\} \\
= & \frac{a}{c} \beta(1-\beta)^{\frac{a+b}{c}} \frac{1}{(A+1)^{k}}\left\{1+\frac{(A+1)^{k}\left(\frac{a+2 b}{c}+1\right) \cdot 1}{(A+2)^{k} \cdot 2} \cdot \frac{\beta(1-\beta)^{\frac{b}{c}}}{1 !}\right. \\
& \left.\left.+\frac{(A+1)^{k}(A+2)^{k}\left(\frac{a+3 b}{c}+1\right)\left(\frac{a+3 b}{c}+2\right) \cdot 2 \cdot 1}{(A+3)^{k}(A+2)^{k} \cdot 3 \cdot 2} \cdot \frac{\beta^{2}(1-\beta)^{\frac{2 b}{c}}}{2 !}+\cdots\right\}, 1 ;(A+2, k), 2 ; \beta(1-\beta)^{\frac{b}{c}}\right] .
\end{aligned}
$$

Note: when $k=1$, the inverse moment of first order is given by

$$
\begin{aligned}
& E(X+A)^{-1} \\
= & \frac{\frac{a}{c} \beta(1-\beta)^{\frac{a+b}{c}}}{A+1}{ }_{3} F_{2}\left[A+1,\left(\frac{a+(n+1) b+c}{c}\right), 1 ; A+2,2 ; \beta(1-\beta)^{\frac{b}{c}}\right] .
\end{aligned}
$$

Let $b=0, k=1$ in theorem 2.2, then inverse moment of first order of the Polya-Eggenberger distribution is

$$
E(X+A)^{-1}=\frac{\frac{a}{c} \beta(1-\beta)^{\frac{a}{c}}}{A+1}{ }_{3} F_{2}\left[A+1,\left(\frac{a+c}{c}\right), 1 ; A+2,2 ; \beta\right] .
$$


Let $b=-1, c=1, k=1$ in theorem 2.2, then inverse moment of first order of the binomial distribution is

$$
E(X+A)^{-1}=\frac{a \beta(1-\beta)^{a-1}}{A+1}{ }_{3} F_{2}\left[A+1,(a-n), 1 ; A+2,2 ; \frac{\beta}{1-\beta}\right] .
$$

Let $c=1, k=1, b=\gamma-1$ in theorem 2.2, then can get the theorem 1 in the [5]

$$
E(X+A)^{-1}=\frac{a \beta(1-\beta)^{a+\gamma-1}}{A+1}{ }_{3} F_{2}\left[A+1, a+(n+1) \gamma-n, 1 ; A+2,2 ; \beta(1-\beta)^{\gamma-1}\right] \text {. }
$$

Let $c \rightarrow 0, \frac{b}{a}=\alpha, \frac{\alpha \beta}{c}=\theta, k=1$ in theorem 2.2 , then inverse moment of first order of the generalized Possion distribution is

$$
E(X+A)^{-1}=\frac{\theta e^{-\theta(1+\alpha)}}{A+1}{ }_{2} F_{2}\left[A+1,1 ; A+2,2 ; \frac{\theta[1+(n+1) \alpha]}{e^{\theta \alpha}}\right] .
$$

Corollary 2.1: Suppose $x$ is a Katz random variable with parameters $\alpha, \beta$, for $\alpha>0,0<\beta<1$. then the inverse moment of $k^{\text {th }}$ order is given by

$$
E(X+A)^{-k}=\frac{a(1-\beta)^{\frac{a}{\beta}}}{(A+1)^{k}}{ }_{3} F_{2}\left[(A+1, k),\left(\frac{a+\beta}{\beta}\right), 1 ;(A+2, k), 2 ; \beta\right] .
$$

where $A \geq 0, n=0,1,2 \cdots$

Proof. Let $b=0, c=\beta$ in theorem 2.2, By definition 3, then

$$
E(X+A)^{-k}=\frac{a(1-\beta)^{\frac{a}{\beta}}}{(A+1)^{k}}{ }_{3} F_{2}\left[(A+1, k),\left(\frac{a+\beta}{\beta}\right), 1 ;(A+2, k), 2 ; \beta\right] .
$$

Note: when $k=1$, the inverse moment of first order is given by

$$
E(X+A)^{-1}=\frac{a(1-\beta)^{\frac{a}{\beta}}}{A+1}{ }_{3} F_{2}\left[A+1,\left(\frac{a+\beta}{\beta}\right), 1 ; A+2,2 ; \beta\right]
$$

Corollary 2.2: Suppose $x$ is a Lagrangian Katz random variable with parameters $a, b, \beta$, for $a>0, b+\beta \geq 0,0<\beta<1$, then the inverse moment of $k^{\text {th }}$ order is given by

$$
\begin{aligned}
& E(X+A)^{-k} \\
& =\frac{a(1-\beta)^{\frac{a+b}{\beta}}}{(A+1)^{k}}{ }_{3} F_{2}\left[(A+1, k),\left(\frac{a+(n+1) b+\beta}{\beta}\right), 1 ;(A+2, k), 2 ; \beta(1-\beta)^{\frac{b}{\beta}}\right]
\end{aligned}
$$

where $A \geq 0, n=0,1,2 \cdots$

Proof. Let $c=\beta$ in theorem 2.2, by definition 4 , then

$$
\begin{aligned}
& E(X+A)^{-k} \\
& =\frac{a(1-\beta)^{\frac{a+b}{\beta}}}{(A+1)^{k}}{ }_{3} F_{2}\left[(A+1, k),\left(\frac{a+(n+1) b+\beta}{\beta}\right), 1 ;(A+2, k), 2 ; \beta(1-\beta)^{\frac{b}{\beta}}\right]
\end{aligned}
$$

Note: when $k=1$, the inverse moment of first order is given by 


$$
E(X+A)^{-1}=\frac{a(1-\beta)^{\frac{a+b}{\beta}}}{A+1}{ }_{3} F_{2}\left[A+1,\left(\frac{a+(n+1) b+\beta}{\beta}\right), 1 ; A+2,2 ; \beta(1-\beta)^{\frac{b}{\beta}}\right]
$$

\section{The Factorial Inverse Moments of Some Discrete Distributions}

In this section, we use generalized hypergeometric series to obtain the inverse factorial moments of some discrete distributions.

Theorem 3.1: Suppose $x$ is a generalized geometric random variable with parameters $\lambda$, for $0<\lambda<1$, then the factorial inverse moment of $k^{\text {th }}$ order is given by

$$
E\left[\prod_{i=1}^{l}(x+i)\right]^{-k}=\frac{(1-\lambda)^{2}}{[(l+1) !]^{k}} F_{1}[(2, k+1) ;(l+2, k) ; \lambda] .
$$

where $l \in Z^{+}$.

Proof. By definition 1, then

$$
\begin{aligned}
E\left[\prod_{i=1}^{l}(x+i)\right]^{k} & =\sum_{x=1}^{\infty} x \lambda^{x-1}(1-\lambda)^{2} \prod_{i=1}^{l}\left(\frac{1}{x+i}\right)^{k} \\
& =\sum_{x=1}^{\infty} x \lambda^{x-1}(1-\lambda)^{2}\left[\frac{x !}{(l+x) !}\right]^{k} \\
& =\frac{(1-\lambda)^{2}}{[(l+1) !]^{k}}\left\{1+\frac{2 \cdot \lambda(2 !)^{k}}{(l+2)^{k}}+\frac{3 \cdot \lambda^{2}(3 !)^{k}}{(l+2)^{k}(l+3)^{k}}+\cdots\right\} \\
& =\frac{(1-\lambda)^{2}}{[(l+1) !]^{k}}\left\{1+\frac{2^{k} \cdot 2}{(l+2)^{k}} \frac{\lambda}{1 !}+\frac{2^{k} \cdot 3^{k} \cdot 3 \cdot 2}{(l+2)^{k}(l+3)^{k}} \cdot \frac{\lambda^{2}}{2 !}+\cdots\right\} \\
& =\frac{(1-\lambda)^{2}}{[(l+1) !]^{k}} F_{1}[(2, k), 2 ;(l+2, k) ; \lambda] \\
& =\frac{(1-\lambda)^{2}}{[(l+1) !]^{k}} F_{1}[(2, k+1) ;(l+2, k) ; \lambda] .
\end{aligned}
$$

Note: when $k=1$, the factorial inverse moment of first order is given by

$$
E\left[\prod_{i=1}^{l}(x+i)\right]^{-1}=\frac{(1-\lambda)^{2}}{(l+1) !}{ }_{1} F_{1}[(2,2) ; l+2 ; \lambda] .
$$

Theorem 3.2: Suppose $x$ is a generalized Polya-Eggenberger of the first kind random variable with parameters $a, b, c, \beta$, for $a>0, c>0, b+c \geq 0,0<\beta<1$, then we have the factorial inverse moment of $k^{\text {th }}$ order is given by

$$
\begin{aligned}
& E\left[\prod_{i=1}^{l}(x+i)\right]^{-k} \\
& =\frac{\frac{a}{c} \beta(1-\beta)^{\frac{a+b}{c}}}{[(l+1) !]^{k}}{ }_{3} F_{2}\left[(2, k),\left(\frac{a+(n+1) b+c}{c}\right), 1 ;(l+2, k), 2 ; \beta(1-\beta)^{\frac{b}{c}}\right] .
\end{aligned}
$$

where $l \in Z^{+}, n \in N$. 
Proof. By definition 2, then

$$
\begin{aligned}
& E\left[\prod_{i=1}^{l}(x+i)\right]^{-k}=\sum_{x=1}^{l} \frac{a}{c} \frac{1}{\left(\frac{a+x b}{c}+x\right)}\left(\frac{a+x b}{c}+x\right) \beta^{x}(1-\beta)^{\frac{a+x b}{c}} \prod_{i=1}^{l}\left(\frac{1}{x+i}\right)^{k} \\
= & \sum_{x=1}^{l} \frac{a}{c} \frac{\left(\frac{a+x b}{c}+x-1\right) !}{x !\left(\frac{a+x b}{c}\right) !} \beta^{x}(1-\beta)^{\frac{a+x b}{c}}\left[\frac{x !}{(l+x)}\right]^{k}, \\
= & \frac{a}{c} \beta(1-\beta)^{\frac{a+x b}{c}} \frac{1}{[(l+1) !]^{k}}\left\{1+\frac{(2 !)^{k}\left(\frac{a+2 b}{c}+1\right)}{(l+2)^{k} \cdot 2 !} \beta(1-\beta)^{\frac{b}{c}}\right. \\
& \left.+\frac{(3 !)^{k}\left(\frac{a+3 b}{c}+1\right)\left(\frac{a+3 b}{c}+2\right)}{(l+2)^{k}(l+3)^{k} \cdot 3 !} \beta^{2}(1-\beta)^{\frac{2 b}{c}}+\cdots\right\} \\
& \frac{\frac{a}{c} \beta(1-\beta)^{\frac{a+b}{c}}}{[(l+1) !]^{k}}{ }_{3} F_{2}\left[(2, k),\left(\frac{a+(n+1) b+c}{c}\right), 1 ;(l+2, k), 2 ; \beta(1-\beta)^{\frac{b}{c}}\right] .
\end{aligned}
$$

Note: when $k=1$, the factorial inverse moment of first order is

$$
E\left[\prod_{i=1}^{l}(x+i)\right]^{-1}=\frac{\frac{a}{c} \beta(1-\beta)^{\frac{a+b}{c}}}{(l+1) !}{ }_{2} F_{1}\left[\left(\frac{a+(n+1) b+c}{c}\right), 1 ; l+2 ; \beta(1-\beta)^{\frac{b}{c}}\right]
$$

Let $b=0, k=1$ in theorem 3.2, then factorial inverse moment of first order of the Polya-Eggenberger distribution is

$$
E\left[\prod_{i=1}^{l}(x+i)\right]^{-1}=\frac{\frac{a}{c} \beta(1-\beta)^{\frac{a}{c}}}{(l+1) !}{ }_{2} F_{1}\left[\left(\frac{a+c}{c}\right), 1 ; l+2 ; \beta\right]
$$

Let $b=-1, c=1, k=1$ in theorem 3.2, then factorial inverse moment of first order of the binomial distribution is

$$
E\left[\prod_{i=1}^{l}(x+i)\right]^{-1}=\frac{a \beta(1-\beta)^{a-1}}{(l+1) !}{ }_{2} F_{1}\left[(a-n), 1 ; l+2 ; \frac{\beta}{1-\beta}\right]
$$

Let $c=1, k=1, b=\gamma-1$ in theorem 3,.2, then can get the theorem 6 in the [5]

$$
E\left[\prod_{i=1}^{l}(x+i)\right]^{-1}=\frac{a \beta(1-\beta)^{a+\gamma-1}}{(l+1) !}{ }_{2} F_{1}\left[a+(n+1) \gamma-n, 1 ; l+2 ; \beta(1-\beta)^{\gamma-1}\right]
$$

Let $c \rightarrow 0, \frac{b}{a}=\alpha, \frac{\alpha \beta}{c}=\theta, k=1$ in theorem 3.2, then factorial inverse moment of first order of the generalized Possion distribution is

$$
E\left[\prod_{i=1}^{l}(x+i)\right]^{-1}=\frac{\theta e^{-\theta(1+\alpha)}}{(l+1) !}{ }_{1} F_{1}\left[1 ; l+2 ; \frac{\theta[1+(n+1) \alpha]}{e^{\theta \alpha}}\right]
$$


Corollary 3.1: Suppose $x$ is a Katz random variable with parameters $\alpha, \beta$, for $\alpha>0,0<\beta<1$. then the factorial inverse moment of $k^{\text {th }}$ order is given by

$$
E\left[\prod_{i=1}^{l}(x+i)\right]^{-k}=\frac{a(1-\beta)^{\frac{a}{\beta}}}{[(l+1) !]^{k}} F_{2}\left[(2, k),\left(\frac{a+\beta}{\beta}\right), 1 ;(l+2, k), 2 ; \beta\right]
$$

where $l \in Z^{+}$.

Proof. Let $b=0, c=\beta$ in theorem 3.2, by definition 3, then

$$
E\left[\prod_{i=1}^{l}(x+i)\right]^{-k}=\frac{a(1-\beta)^{\frac{a}{\beta}}}{[(l+1) !]^{k}} F_{2}\left[(2, k),\left(\frac{a+\beta}{\beta}\right), 1 ;(l+2, k), 2 ; \beta\right] .
$$

Note: when $k=1$, the factorial inverse moment of first order is given by

$$
E\left[\prod_{i=1}^{l}(x+i)\right]^{-1}=\frac{a(1-\beta)^{\frac{a}{\beta}}}{(l+1) !}{ }_{2} F_{1}\left[\left(\frac{a+\beta}{\beta}\right), 1 ; l+2 ; \beta\right] .
$$

Corollary 3.2: Suppose $X$ is a Lagrangian Katz random variable with parameters $a, b, \beta$, for $a>0, b+\beta \geq 0,0<\beta<1$, then the factorial inverse moment of $k^{\text {th }}$ order is given by

$$
\begin{aligned}
& E\left[\prod_{i=1}^{l}(x+i)\right]^{-k} \\
& =\frac{a(1-\beta)^{\frac{a+b}{\beta}}}{[(l+1) !]^{k}} F_{2}\left[(2, k),\left(\frac{a+(n+1) b+\beta}{\beta}\right), 1 ;(l+2, k), 2 ; \beta(1-\beta)^{\frac{b}{\beta}}\right] .
\end{aligned}
$$

where $l \in Z^{+}, n \in N$.

Proof. Let $c=\beta$ in theorem 3.2, by definition 4 , then

$$
\begin{aligned}
& E\left[\prod_{i=1}^{l}(x+i)\right]^{-k} \\
& =\frac{a(1-\beta)^{\frac{a+b}{\beta}}}{[(l+1) !]^{k}} F_{2}\left[(2, k),\left(\frac{a+(n+1) b+\beta}{\beta}\right), 1 ;(l+2, k), 2 ; \beta(1-\beta)^{\frac{b}{\beta}}\right] .
\end{aligned}
$$

Note: when $k=1$, the inverse factorial moment of first order is given by

$$
E\left[\prod_{i=1}^{l}(x+i)\right]^{-1}=\frac{a(1-\beta)^{\frac{a+b}{\beta}}}{(l+1) !}{ }_{2} F_{1}\left[\left(\frac{a+(n+1) b+\beta}{\beta}\right), 1 ; l+2 ; \beta(1-\beta)^{\frac{b}{\beta}}\right] .
$$

\section{References}

[1] Stephan, F.F. (1945) The Expected Value and Variance of the Reciprocal and Other Negative Powers of a Positive Bernoulli Variate. Annals of Mathematical Statistics, 16, 50-61. https://doi.org/10.1214/aoms/1177731170

[2] Grab, E.L. and Savage, I.R. (1954) Tables of the Expected of 1/x for Positive Bernoulli and Poisson Variable. Journal of American Statistical Association, 49, 167-177.

[3] Govindarajulu, Z. Recurrence Relation for the Inverse Moments of the Positive Binomial Variable. Journal of American Statistical Association, 58, 468-473. https://doi.org/10.1080/01621459.1963.10500859 
[4] Chao, M.T. and Strawderman, W.E. (1972) Negative Moments of Positive Random Variables. Journal of the American Statistical Association, 67, 429-431. https://doi.org/10.1080/01621459.1972.10482404

[5] Wang, C.Y. and Wuyungaowa (2015) Recurrence Relation and Accurate Value on Inverse Moment of Discrete Distributions. Journal of Probability and Statistics. https://doi.org/10.1155/2015/972035

[6] Znidaric, M. (2005) Asymptotic Expansion for Inverse Moments of Binomial and Poisson Distribution. Statistics Theory [math.ST].

Submit or recommend next manuscript to SCIRP and we will provide best service for you:

Accepting pre-submission inquiries through Email, Facebook, LinkedIn, Twitter, etc. A wide selection of journals (inclusive of 9 subjects, more than 200 journals)

Providing 24-hour high-quality service

User-friendly online submission system

Fair and swift peer-review system

Efficient typesetting and proofreading procedure

Display of the result of downloads and visits, as well as the number of cited articles Maximum dissemination of your research work

Submit your manuscript at: http://papersubmission.scirp.org/

Or contact jamp@scirp.org 